\title{
A dependência da nicotina associada ao uso de álcool e outras substâncias psicoativas
}

\section{The nicotine dependence associated with alcohol use and other psychoactive substance}

\author{
Márcia Regina Pizzo de Castro ${ }^{1}$; Sandra Odebrecht Vargas Nunes²; Danilo \\ Donizete de Faria ${ }^{3}$; Carlos Eduardo Bobroff da Rocha ${ }^{4}$; Rafaela Serra Bacchi ${ }^{5}$
}

Resumo

\begin{abstract}
Analisar a associação entre a dependência de nicotina com o uso de álcool, outras substâncias psicoativas e transtorno depressivo. Os tabagistas foram recrutados a partir do Centro de Referência de Abordagem e Tratamento do Tabagismo no Hospital de Clínicas da Universidade Estadual de Londrina (AHC/UEL). Todos os participantes foram informados e assinaram o termo de consentimento livre e esclarecido aprovado pelo Comitê de Ética em Pesquisa da Universidade Estadual de Londrina. Os instrumentos foram: questionário estruturado, teste de triagem do envolvimento com álcool, tabaco e outras substâncias (ASSIST v 3.0), teste de Fargerström de Dependência de Nicotina (FTND) e critérios diagnósticos para pesquisa de transtorno depressivo, da Organização Mundial da Saúde. Os tabagistas apresentaram as seguintes características sócio-demográficas: predomínio do sexo feminino, e média de idade de 47 anos, com capacidade para atividades domésticas e trabalho. A média de idade de início do uso do tabaco foi de 16 anos. O teste de Fagerström apresentou uma pontuação média de seis, tanto para tabagistas com e sem uso de substâncias psicoativas, A relação encontrada entre a depressão grave e o uso de substâncias psicoativas foi significativa. Este estudo evidenciou uma associação entre o uso do tabaco, de outras substâncias psicoativas e transtorno depressivo. Os profissionais da saúde deveriam identificar subgrupos de fumantes adultos com associação de depressão e uso de substâncias psicoativas, e promover uma intervenção em ambas as comorbidades para maior efetividade do abandono do tabaco. Palavras-chave: Dependência de nicotina. Uso de álcool. Uso de substâncias psicoativas. Transtorno depressivo.
\end{abstract}

\begin{abstract}
To examine an association between nicotine dependence with alcohol, other psychoactive use, and depressive disorder. Smokers were recruited from Centro de Referência de Abordagem e Tratamento do Tabagismo at the Hospital das Clínicas da Universidade Estadual de Londrina (AHC/ UEL). All subjects were informed and gave then written consent for the research as approved by the Ethics Research Committee of Universidade Estadual de Londrina. The measures used were: structured questionnaire,
\end{abstract}

1 Coordenação do Centro de Cessação do Tabaco do Ambulatório do Hospital de Clínicas da Universidade Estadual de Londrina, mpizzo@sercomtel.com.br.

2 Departamento de Clínica Médica/ Centro de Ciências da Saúde da Universidade Estadual de Londrina, sandranunes@sercomtel. com.br.

3 Acadêmico do Curso de Medicina da Universidade Estadual de Londrina, bolsista de iniciação científica- PIBIC./CNPq, danilo_ faria84@hotmail.com

4 Acadêmico do Curso de Medicina da Universidade Estadual de Londrina, colaborador de iniciação científica - PIBIC./CNPq, carlospidgeot@yahoo.com.

5 Acadêmica do curso de enfermagem da Universidade Norte do Paraná, rafaela.bacchi@yahoo.com.br 
alcohol, smoking, and psychoactive substance involvement screening test (ASSIST v 3.0), the Fagerström test for Nicotine Dependence (FTND), and the Diagnostic Interview for Research on Depressive disorder of the World Health Organization. Smokers presented the following socio-demographic characteristics: prevalence of the female sex and mean age of 47 years old with capacity for domestic activities and work. The mean age of onset of cigarette use for smokers was 16 years old. Fagerström's test presented a medium punctuation of 6 , so much for users of substances psicoativas, as for the ones that they don't use them. Relationship between serious depression and the of psychoactive substances use was relevant for the research. This study evidenced an association among the use of the tobacco and other psychoactive substances, and depressive disorder. The health professional in smoking cessation intervention would be to identify subgroups of adult smokers, associated with depression, psychoactive substance use, and promote an intervention in both comorbidities and larger effectiveness of the smoking cessation.

Keywords: Nicotine dependence. Alcohol use. Psychoactive substances use. Depressive disorder.

\section{Introdução}

Tabaco, álcool e drogas ilícitas estão entre os 20 fatores de risco para doenças. Tal fato decorre do encargo global devido às doenças associadas ao uso do tabaco, e este encargo é mensurado como o número de anos que são gastos ao conviver com a doença (anos de incapacidade de adaptação da vidaDALY) É estimado que o tabaco seja responsável por $8,8 \%$ de todas as mortes, e por $4,1 \%$ da DALY. Com relação ao álcool, estima-se que seja responsável por 3,2 \% das mortes, e por $4,0 \%$ da DALY. Drogas ilícitas são responsáveis por $0,4 \%$ das mortes, e por $0,8 \%$ da DALY. Mundialmente, há uma crescente tendência de as pessoas utilizarem múltiplas substâncias em conjunto e em diferentes momentos, o que favorece o aumento dos riscos (WORLD HEALTH ORGANIZATION, 2003).

O consenso de tratamento de dependência de nicotina publicado pela American Psychiatric Association (1996), listou algumas ações que são essenciais, e estas incluem incentivar o diagnóstico apropriado e o tratamento adequado da dependência da nicotina, visto esta ser uma condição de comorbidade relacionada com outras desordens psiquiátricas.

Preditores psiquiátricos que incentivam o início no uso do cigarro incluem o consumo abusivo de álcool e de outras drogas psicoativas, bem como a presença de transtornos de déficit de atenção e de sintomas depressivos. Em vista disso, o tabagismo precede a idade normal de inicio de muitas doenças

\section{(AMERICAN PSYCHIATRIC ASSOCIATION,} 1996).

Dependentes de nicotina têm maior probabilidade de sofrer de transtornos psiquiátricos concomitantes de que a população geral. Cerca de 60 a 95\% dos indivíduos apresentam dependência de nicotina concomitante com outras substâncias psicoativas e o tratamento de uso de substâncias psicoativas coexistentes é um desafio. O tratamento de transtornos psiquiátricos coexistentes inclui intervenções psicossociais e /ou farmacológico. A falta de adesão pode ter muitos fatores, incluindo comprometimento cognitivo, medo do paciente de interações medicamentosas prescrita e as substâncias de abuso, medo que a própria medicação prescrita seja prejudicial, mudança na motivação e falta de apoio (AMERICAN PSYCHIATRIC ASSOCIATION, 2008).

Para identificar fumantes com comorbidades associadas a outras substâncias psicoativas, utilizase o ASSIST (Alcohol, Smoking, and Substance Involvement Screen Test), que é um teste de triagem breve para detectar o uso nocivo ou de risco de álcool, cigarro e drogas ilícitas. O ASSIST é direcionado para profissionais de atenção primária à saúde para o uso de suas rotinas de trabalho (WORLD HEALTH ORGANIZATION, 2003).

O uso de substâncias psicoativas (tabaco, álcool, maconha, cocaína, estimulantes, inalantes, sedativos, hipnóticos, alucinógenos, opióides) pode induzir sensações de prazer aos usuários. Teorias acerca da 
dependênciatêm, principalmente, sidodesenvolvidas a partir de evidências neurobiológicas e de dados de estudos dos mecanismos do comportamento e da memória (CAMI; FARRE, 2003). O reforço positivo no uso de psicoativos ocorre por ativação das vias de recompensa da dopamina do sistema mesocorticolímbico do cérebro (NOBLE et al., 1994). O aumento da dopamina sináptica no núcleo accumbens é o mecanismo de recompensa para muitas drogas de abuso (BERRETTINI; LERMAN, 2005).

Em estudos anteriores, foi estabelecido que fumantes com uma história de desordens mentais têm uma baixa taxa de sucesso na cessação de fumar (SCHMITZ; KRUSE; KUGLER, 2003).

Cerca de 15 a $20 \%$ dos que fumam excessivamente apresentam comorbidades com dependência e abuso do álcool e 25 a $40 \%$ dos fumantes que procuram tratamento têm um passado históricodedepressão(AMERICANPSYCHIATRIC ASSOCIATION, 1996). Cerca de 50\% dos pacientes psiquiátricos fumam, em contraste com os $25 \%$ da população geral. Além disso, $50 \%$ da população geral conseguem parar de fumar, enquanto somente $15 \%$ dos pacientes psiquiátricos o fazem (LEMOS; GICLIOTTI, 2006).

O uso de várias drogas é freqüente nos indivíduos com dependência, e muitos preenchem estes critérios. Desordens psiquiátricas estão associadas com um aumento do risco de abuso. Um diagnóstico dual (abuso de substância e desordem mental) tem implicações desfavoráveis para o tratamento e para suas conseqüências (CAMI; FARRE, 2003).

O objetivo deste estudo foi conhecer as características sócio-demográficas, hábito tabágico de fumantes de um Centro de Referência de Abordagem e Tratamento do Tabagismo da UEL e analisar a dependência do tabaco e sua associação com uso de substâncias psicoativas e transtorno depressivo.

\section{Casuística e método}

O delineamento da pesquisa foi um estudo transversal. A amostra foi de conveniência de tempo e lugar, composta por 123 tabagistas ambulatoriais motivados no desejo de parar de fumar. Fumantes de cigarros, de ambos os sexos, entre as idades de $18 \mathrm{e}$ de 65 anos, foram recrutados entre março de 2006 , e março de 2007 em um Centro de Referência de Abordagem e Tratamento do Tabagismo no Hospital de Clínicas da Universidade Estadual de Londrina (AHC/UEL) sendo credenciado pelo Ministério da Saúde, de acordo com a Portaria SAS/MS 442/04 (MINISTÉRIO DA SAÚDE, 2004).

Todos os tabagistas tiveram a intenção de se tratarem por pelo menos um ano, fumavam mais de 10 cigarros por dia, e receberam terapia cognitivocomportamental para a cessação do tabagismo. De acordo com a necessidade individual, alguns tabagistas fizeram terapias combinadas de fármacos (antidepressivos e terapias de reposição de nicotina).

Foram excluídos tabagistas portadores de déficit cognitivos, de retardo mental, e de demência, pelas dificuldades na abordagem comportamental.

Todos os participantes foram convidados para uma orientação sobre o estudo, e o termo de consentimento livre e esclarecido assinado foi obtido. O Comitê de Ética em Pesquisa da Universidade Estadual de Londrina aprovou a pesquisa.

Levaram-se em conta as informações referentes às características sócio-demográficas, e à história do hábito tabágico. O Teste de Fagerstöm para a dependência de nicotina (FTND) foi utilizado e a partir de seus seis itens determinou-se o comportamento no ato de fumar, e com isso, estabeleceu-se o grau de dependência de nicotina por meio das médias obtidas em seu questionário. Embora o escore máximo do teste de fagerström seja 10, um escore cuja pontuação seja maior que 5 indica, provavelmente, a dependência de nicotina (BERRETTINI; LERMAN, 2005).

O Teste de Seleção Envolvendo Álcool, Cigarro e Substâncias Psicoativas (ASSIST v 3.0) trata-se de um questionário para rastreamento de pessoas que 
fazem uso de substâncias psicoativas, que abrange: tabaco, álcool, canabinóides, cocaína, estimulantes do tipo anfetamina, sedativos, alucinógenos, inalantes, opióides e outras drogas A adaptação transcultural para a língua portuguesa foi realizada por Henrique et al (2004).

Os critérios diagnósticos para pesquisa de transtorno depressivo estão de acordo com a Décima Revisão da Classificação Internacional de Doenças e Problemas de Saúde Relacionados (CID10), segundo a ORGANIZAÇÃO MUNDIAL DE SAÚDE (1998).

Os dados foram armazenados e analisados no Programa Epi Info versão 3.3.2.. O tabaco associado ou não ao álcool e a outras substâncias psicoativas foram comparados em variáveis sóciodemográficas e clínicas. Para análise dos dados foi empregado o cálculo das médias, desvio padrão, e freqüências bruta e percentual, apresentadas em tabelas. A comparação entre os grupos em relação às variáveis sócio-demográficas categorizadas foi analisada pelo teste do Qui-quadrado $\left(\chi^{2}\right)$, ou o teste Exato de Fisher (freqüência esperada $<5$ ). A comparação entre os grupos em relação às variáveis clínicas foi analisada pelo teste de Mann-Whitney. Foi adotado o nível de significância de 5\% em todos os testes estatísticos. Diferenças entre os grupos foram consideradas como sendo estatisticamente significativo quando $\mathrm{p}<0,05$.

\section{Resultados}

A amostra foi composta por 123 fumantes, com média de idade de 45 anos para fumantes com uso de substâncias psicoativas e 47 anos para fumantes sem uso de substâncias psicoativas. A busca para o tratamento foi maior entre as mulheres fumantes com e sem uso de substâncias psicoativas comparadas aos homens. Não se obteve diferenças significativas sem relação em relação ao Fagerström, idade de início, anos de vida fumado, conforme apresentado na Tabela 1 .

Tabela 1. Características sócio-demográficas e o hábito tabágico em fumantes com e sem uso de substâncias psicoativas recrutados do AHC/UEL.

\begin{tabular}{|c|c|c|c|}
\hline Características & $\begin{array}{l}\text { Fumantes com uso de } \\
\text { substâncias psicoativas } \\
\qquad(\mathrm{n}=51)\end{array}$ & $\begin{array}{l}\text { Fumantes sem uso de } \\
\text { substâncias psicoativas } \\
\qquad(\mathrm{n}=72)\end{array}$ & Valor de $\mathrm{p}$ \\
\hline Sexo & & & $0,6804^{*}$ \\
\hline sexo masculino (n e \%) & $21(43,8 \%)$ & $27(56,3 \%)$ & \\
\hline sexo feminino (n e \%) & $30(40,00 \%)$ & $45(60,00 \%)$ & \\
\hline Idade & & & $0,3850^{+}$ \\
\hline média \pm desvio padrão & $45,03 \pm 9,15$ & $47,11 \pm 8,62$ & \\
\hline mediana e interquartis & $47(41-52)$ & $46,5(41,5-52,5)$ & \\
\hline Fagerström & & & $0,5127^{+}$ \\
\hline média \pm desvio padrão & $5,90 \pm 2,56$ & $5,71 \pm 2,12$ & \\
\hline mediana e interquartis & $6(4-8)$ & $6(5-7)$ & \\
\hline Idade de ínicio & & & $0,6854^{+}$ \\
\hline média \pm desvio padrão & $16 \pm 4,77$ & $16,25 \pm 5,14$ & \\
\hline mediana e interquartis & $15(13-19)$ & $15(14-17)$ & \\
\hline Anos de vida fumado & & & $0,6937^{+}$ \\
\hline média \pm desvio padrão & $28,19 \pm 8,21$ & $29,23 \pm 9,20$ & \\
\hline mediana e interquartis & $30(23-34)$ & $30(22-35)$ & \\
\hline
\end{tabular}


Na Tabela 2 não se obteve a resposta de dois fumantes com relação às variáveis doenças que afaste/incapacite trabalho, de quatro fumantes em relação à história de transtorno mental na família e de três fumantes em relação ao transtorno depressivo.
Nos fumantes com uso de substâncias psicoativas houve diferenças significativas na incapacidade laboral $(\mathrm{p}=0,047)$, assim como, houve diferenças significativas em mais transtorno depressivo $(\mathrm{p}=0,009)$.

Tabela 2. Incapacidade laboral, história familiar de transtorno mental e transtorno depressivo, em fumantes com e sem uso de substâncias psicoativas recrutados do AHC/UEL.

\begin{tabular}{|c|c|c|c|}
\hline Características & $\begin{array}{l}\text { Fumantes com uso de } \\
\text { substâncias psicoativas } \\
\qquad(\mathrm{n}=51)\end{array}$ & $\begin{array}{l}\text { Fumantes sem uso de } \\
\text { substâncias psicoativas } \\
\qquad(\mathrm{n}=72)\end{array}$ & Valor de $\mathrm{p}$ \\
\hline Doença que afastel & & & $0,0470^{*}$ \\
\hline $\begin{array}{l}\text { incapacite trabalho } \\
\operatorname{sim}(\mathrm{n} \mathrm{e} \%) \\
\text { não }(\mathrm{n} \mathrm{e} \%)\end{array}$ & $\begin{array}{c}20(39,21 \%) \\
31(62 \%)\end{array}$ & $\begin{array}{c}15(21,4 \%) \\
55(64 \%)\end{array}$ & \\
\hline $\begin{array}{l}\text { História de transtorno } \\
\text { mental na família } \\
\text { sim (n e \%) } \\
\text { não (n e \%) }\end{array}$ & $\begin{array}{l}30(58,8 \%) \\
21(41,2 \%)\end{array}$ & $\begin{array}{l}35(51,5 \%) \\
33(48,5 \%)\end{array}$ & $0,4252^{*}$ \\
\hline $\begin{array}{l}\text { Gravidade da depressão } \\
\text { ausente (n e \%) } \\
\text { leve(n e \%) } \\
\text { moderado(n e \%) } \\
\text { grave(n e \%) }\end{array}$ & $\begin{array}{c}21(41,2 \%) \\
11(21,6 \%) \\
8(15,7 \%) \\
11(21,6 \%)\end{array}$ & $\begin{array}{c}39(56,5 \%) \\
13(18,8 \%) \\
15(21,7 \%) \\
2(2,9 \%)\end{array}$ & $0,0094^{*}$ \\
\hline
\end{tabular}

*Teste de qui-quadrado

$\mathrm{Na}$ Tabela 3 encontraram-se diferenças significativas entre gênero em fumantes que fazem abuso do álcool em comparação aos fumantes não consumidores de álcool $(p=0,0005)$. A média de idade foi de 45 anos para fumantes com uso abusivo de álcool e 50 anos para fumantes sem uso abusivo de álcool. Com relação a variável anos de vida fumado, encontrou-se maior anos fumados para tabagistas sem uso abusivo de álcool comparado aos tabagistas com uso abusivo de álcool. Não houve diferença significativa na gravidade de dependência de nicotina (FTND) e na idade de início do consumo do tabaco.

Na Tabela 4 não se obteve a resposta de três fumantes com relação a incapacidade laboral, quatro em relação a história de transtorno mental na família, e dois em relação ao grau de depressão. Não houve diferenças significativas em fumantes com e sem uso abusivo de álcool em relação a incapacidade laboral, história familiar de transtornos mentais e transtorno depressivo. 
Tabela 3. Características sócio-demográficas e o hábito tabágico em fumantes com e sem uso de álcool recrutados do AHC/UEL.

\begin{tabular}{|c|c|c|c|}
\hline Características & $\begin{array}{l}\text { Fumantes com uso de } \\
\text { álcool }(n=99)\end{array}$ & $\begin{array}{l}\text { Fumantes sem uso de álcool } \\
\qquad(\mathrm{n}=24)\end{array}$ & Valor de $\mathrm{p}$ \\
\hline $\begin{array}{l}\text { Sexo } \\
\text { sexo masculino (n e \%) } \\
\text { sexo feminino (n e \%) }\end{array}$ & $\begin{array}{l}46(95,8 \%) \\
53(70,7 \%)\end{array}$ & $\begin{array}{c}2(4,2 \%) \\
22(91,7 \%)\end{array}$ & $0,0005^{*}$ \\
\hline $\begin{array}{l}\text { Idade } \\
\text { média } \pm \text { desvio padrão } \\
\text { mediana e interquartis }\end{array}$ & $\begin{array}{c}45,25 \pm 9,01 \\
46(40-52)\end{array}$ & $\begin{array}{l}50,37 \pm 6,98 \\
50(46-54)\end{array}$ & $0,0159^{+}$ \\
\hline $\begin{array}{l}\text { Fagerström } \\
\text { média } \pm \text { desvio padrão } \\
\text { mediana e interquartis }\end{array}$ & $\begin{array}{c}5,70 \pm 2,25 \\
5(4-7)\end{array}$ & $\begin{array}{c}6,19 \pm 2,58 \\
7(4-8)\end{array}$ & $0,2715^{+}$ \\
\hline $\begin{array}{l}\text { Idade de ínicio } \\
\text { média } \pm \text { desvio padrão } \\
\text { mediana e interquartis }\end{array}$ & $\begin{array}{c}16,01 \pm 4,45 \\
15(14-18)\end{array}$ & $\begin{array}{c}16,73 \pm 6,87 \\
15(12-20)\end{array}$ & $0,6997^{+}$ \\
\hline $\begin{array}{l}\text { Anos de vida fumado } \\
\text { média } \pm \text { desvio padrão } \\
\text { mediana e interquartis }\end{array}$ & $\begin{array}{c}27,82 \pm 8,98 \\
30(20-34)\end{array}$ & $\begin{array}{c}33 \pm 6,50 \\
31(30-36)\end{array}$ & $0,0108^{+}$ \\
\hline
\end{tabular}

*Teste de qui-quadrado + +Teste de Mann-Whitney

Tabela 4. Incapacidade laboral, história familiar de transtorno mental e transtorno depressivo em fumantes com e sem uso de álcool recrutados do AHC/UEL.

\begin{tabular}{|c|c|c|c|}
\hline Características & $\begin{array}{l}\text { Fumantes com uso de álcool } \\
\qquad(\mathrm{n}=99)\end{array}$ & $\begin{array}{l}\text { Fumantes sem uso de álcool } \\
\qquad(\mathrm{n}=24)\end{array}$ & Valor de $\mathrm{p}$ \\
\hline \multicolumn{4}{|l|}{$\begin{array}{l}\text { Doença que afastel } \\
\text { incapacite trabalho }\end{array}$} \\
\hline $\operatorname{sim}($ n e $\%)$ & $29(29,3 \%)$ & $5(23,8 \%)$ & \multirow{2}{*}{$0,6125^{*}$} \\
\hline não(n e \%) & $70(70,7 \%)$ & $16(76,2 \%)$ & \\
\hline \multicolumn{4}{|l|}{ História de transtorno } \\
\hline mental na família & & & \multirow{3}{*}{$0,3433^{*}$} \\
\hline $\operatorname{sim}($ n e $\%)$ & $56(56,6 \%)$ & $9(45,00 \%)$ & \\
\hline não(n e \%) & $43(43,4 \%)$ & $11(55,00 \%)$ & \\
\hline \multicolumn{4}{|l|}{ Gravidade da depressão } \\
\hline ausente(n e \%) & $52(53,1 \%)$ & $8(36,4 \%)$ & \multirow{4}{*}{$0,1122^{*}$} \\
\hline leve(n e \%) & $21(21,4 \%)$ & $3(13,6 \%)$ & \\
\hline moderado(n e \%) & $17(17,3 \%)$ & $6(27,3 \%)$ & \\
\hline grave(n e \%) & $8(8,2 \%)$ & $5(22,7 \%)$ & \\
\hline
\end{tabular}

*Teste de qui-quadrado 


\section{Discussão}

No presente estudo, a média de idade de início do tabagismo foi de 16 anos. Alguns estudos relataram que a média de idade de início do tabagismo está entre 13 e 14 anos e que o início mais precoce está associado a maior gravidade da dependência de tabaco e de problemas a ela associados (AMERICAN PSYCHIATRIC ASSOCIATION, 2008; MARQUES et al., 2001). Os tabagistas que começam a fumar na adolescência, 50\% deles morrem prematuramente na meia idade, perdendo cerca de 20 a 25 anos de expectativa de vida em comparação aos não fumantes. $\mathrm{O}$ risco é maior naqueles que começam a fumar regularmente na adolescência (FAGERSTRÖM, 1978).

Embora o maior benefício da cessação de fumar seja obtido na juventude, parar de fumar na meiaidade evita muitos fatores de riscos concomitantes. Deste modo, 15 anos após deixar de fumar torna menor o risco de morte dos ex-fumantes, se comparado com o risco daqueles que nunca fumaram (FARGERSTRÖM, 2002).

Embora a população pesquisada tenha iniciado o uso de tabaco na adolescência, verificou-se que a média de idade de procura de tratamento foi de 47 anos. As pessoas que deixam de fumar antes dos 50 anos de idade apresentam uma redução de $50 \%$ do risco de morte por doenças relacionadas ao tabagismo após 16 anos de abstinência (BRASIL, 2001).

Em nossa casuística, as mulheres buscaram mais tratamento no Centros de Referência de Abordagem e Tratamento do Tabagismo no AHC/UEL., apesar dos estudos relatarem que a prevalência de dependência de tabaco ao longo da vida é de do sexo masculino. Em uma amostra de 42.517 pessoas, sobre a prevalência do tabagismo em uma população acima de 12 anos, na cidade de Londrina, Paraná, revelou um índice próximo de $41,3 \%$ e $22,79 \%$, respectivamente para homens e para mulheres (NUNES et al.,1999) Fumantes masculinos têm maiores escores de dependência à nicotina do que fumantes femininos (FAGERSTRÖM, 1996).
Verificou-se uma grande prevalência de familiares biológicos especialmente em primeiro grau com dependências de tabaco. Um estudo também demonstrou que a dependência do tabaco tem um caráter familial, visto que, familiares em primeiro grau de dependentes de tabaco têm uma freqüência significativamente maior de dependência do que em parentes de probandos não dependentes de tabaco (NUNES et al. 1999).

A maior comorbidade relacionada ao tabagismo e consumo de substâncias psicoativas foi a depressão. Importante também foi a maior ocorrência de depressão grave naqueles que utilizam outras substâncias psicoativas em relação aos que não as utilizam Há uma relação recíproca de fumantes terem mais depressão do que os não fumantes, e os depressivos serem mais fumantes do que os não fumantes. Pacientes depressivos têm aumento do risco de serem dependentes de nicotina, e a associação de depressão e tabaco aumenta duas vezes o risco de doenças cardiovasculares (GLASSMAN, et al., 2001).

No estudo observou-se que o escore para dependência de nicotina por meio do teste de Fagerström (FTND) foi aproximadamente 6. Fumantes com mais forte dependência procuram mais ajuda para cessação do tabaco em relação ao total de fumantes. Fumantes com maiores escores de dependência à nicotina têm menos sucesso em abandonar o tabagismo (FAGERSTRÖM, 1996).

Observou-se também que a comorbidade do tabaco relacionada à depressão e uso de substâncias psicoativas foi estatisticamente significativa. Pacientes depressivos têm maiores risco de fumar e usar outras substâncias psicoativas, e isto está associado a maiores incapacidades e piores resultados de tratamento, em decorrência da depressão exacerbar os afetos negativos encontrados durante a tentativa de abster-se de uma ou ambas drogas.n (AIT-DAOUD et al., 2006). Os pacientes depressivos com comorbidade com tabaco e uso de substâncias psicoativas tiveram estatisticamente maiores incapacidades profissionais. 
O consumo de tabaco também está associado a outras dependências como álcool, maconha, ansiolíticos. Em nosso estudo, a associação entre fumantes de ambos os sexos e o consumo de álcool foi significativa ( $p=0,0005)$. A comorbidade álcool e tabaco multiplica o risco de câncer oral, de laringe, e de esôfago (PELUCCHI et al., 2006). Tal fato reforça a necessidade de os profissionais de saúde em avaliar outras dependências de substâncias psicoativas (HENRIQUE et al., 2004).

Cerca de $50 \%$ dos pacientes psiquiátricos fumam, em contraste com os $25 \%$ da população geral. Também 50\% da população geral conseguem parar de fumar, enquanto somente $15 \%$ dos pacientes psiquiátricos o fazem. Fumantes depressivos percebem o cigarro mais prazeroso do que aqueles sem comorbidades. Este acaba por ser um fator que mina a motivação deste grupamento de tabagistas para deixar de fumar (LEMOS; GIGLIOTTI, 2006).

\section{Considerações finais}

Para abordagem e tratamento do fumante avaliaram-se as características sócio-demográficas, história de transtorno mental na família, idade de início, gravidade da dependência de nicotina, história familial e incapacidade laboral. Os fumantes dos centros de atenção primária deste estudo eram em sua maioria do sexo feminino, de meia idade, com o início do consumo do tabaco aos 16 anos de idade, sem doenças terminais e incapacitantes para atividades laborais, com possibilidades de prevenção de morbidade e mortalidade. Os pacientes com consumo de álcool associado tiveram maior risco de depressão grave.

Os resultados do Ambulatório de Abordagem e Tratamento do Tabagismo na rede SUS indicam que os fumantes iniciaram o consumo na adolescência, mas procuraram tratamento na meia idade, diferiam na gravidade de dependência à nicotina, tinham familiares tabagistas, tiveram comorbidades mentais.
A incapacidade laboral ocorreu entre tabagistas com uso de substãncias psicoativas.

O Tratamento de Cessação do Tabaco deverá considerar o grupo de pacientes tabagistas com comorbidades psiquiátricas, com o consumo alcoólico e de outras substâncias psicoativas, e com depressão grave considerada os mais refratários ao tratamento e com maior dificuldade na prevenção de mortalidade e de morbidade.

\section{Agradecimentos}

À Fundação Araucária, ao Programa de Pesquisa em Ciência e Tecnologia para o SUS, pelo apoio para implantar o Centro de Referência de Abordagem e Tratamento de Fumantes no Hospital de Clínicas da Universidade Estadual de Londrina (AHC/UEL). Ao PIBIC/CNPq ao apoio aos alunos de iniciação científica

\section{Referências}

AIT-DAOUD, N.; LYNCH, W. J.; PENBERTHY, J. K.; BRELAND, A. B.; MARZANI-NISSEN, G. R.; JOHNSON, B. A. Treating smoking dependence in depressed alcoholics. Alcohol Research \& Health, Rockville, v. 29, n. 3, p. 213-219, 2006.

AMERICAN PSYCHIATRIC ASSOCIATION. Practice guideline for the treatment of patients with nicotine dependence. The American Journal of Psychiatry, Arlington, v. 153, Suppl. 10, p. 1-31, Oct. 1996.

- Referência rápida às diretrizes para o tratamento de transtornos psiquiátricos: compêndio 2006. Porto Alegre: Artmed, 2008.

BERRETTINI, W. H.; LERMAN, C. E. Pharmacotherapy and pharmacogenetics of nicotine dependence. The American Journal of Psychiatry; Arlington, v. 162, n. 8, p. 1441-1451, Aug. 2005.

BRASIL. Ministério da Saúde. Instituto Nacional de Câncer. Abordagem e tratamento do fumante. Rio de Janeiro: INCA, 2001.

BRASIL. Ministério da Saúde. Secretaria de Atenção à Saúde. Portaria SAS/MS 442/04 de 13 de agosto de 2004. Aprova o plano de implantação da abordagem e tratamento do tabagismo no SUS. Disponível em: $<\mathrm{http}: / /$ 
dtr2001.saude.gov.br/sas/PORTARIAS/Port2004/PT442.htm>. Acesso em: 6 abr. 2007.

CAMI, J; FARRE, M. Mechanisms of disease: drug addiction. The New England Journal of Medicine, Boston, v. 349, n. 10, p. 975-986, Sep. 2003.

FAGERSTRÖM, K. O.; KUNZE, M.; SCHOBERBERGER, R.; BRESLAU, N.; HUGHES, J. R.; HURT, R. D.; PUSKA, P.; RAMSTROM, L.; ZATONSKI, W. Nicotine dependence versus smoking prevalence: comparisons among countries and categories of smokers. Tobacco Control, London, v. 5, n. 1, p. 52$56,1996$.

FAGERSTRÖM, K. O. Measuring degree of physical dependence to tobacco smoking with reference to individualization of treatment. Addict. Behav., Oxford, v. 3, n. 3/4, p. 235-241, 1978.

FARGERSTRÖM, K. The epidemiology of smoking: health consequences and benefits of cessation. Drugs, Auckland, v. 62, Suppl. 2, p. 1-9, 2002.

GLASSMAN, A. H.; COVEY, L ., STETNER, F., RIVELLI S. Smoking cessation and the course of major depression: a follow up study. Lancet, London, v. 357, n. 9772, p. 1929-1932, Jun. 2001.

HENRIQUE, I. F., MICHELI, D.; LACERDA, R. B.; LACERDA, L. A.; FORMIGONI, M. L. O. S. Validação da versão brasileira do teste de triagem do envolvimento com álcool, cigarro e outras substâncias (ASSIST). Revista da Associação Médica Brasileira, São Paulo, v. 50, n. 2, p. 199-206, abr./jun. 2004.

LEMOS, T; GIGLIOTTI, A. Tabagismo e comorbidades psiquiátricas. In: GIGLOTTI, A.; PRESMAN S. Atualização no tratamento do tabagismo. Rio de Janeiro: ABP Saúde, 2006. p. 53-70.

MARQUES, A. C. P. R.; CAMPANA, A.; GIGLIOTTI, A. P.; LOURENÇO, M. T. C.; FERREIRA, M. P.; LARANJEIRA, R. Consenso sobre o tratamento da dependência de nicotina. Revista Brasileira de Psiquiatria, São Paulo, v. 23, n. 4, p. 200-214, dez. 2001.

NOBLE, E. P.; ST JEOR, S. T.; RITCHIE, T.; SYNDULKO, K.; ST JEOR, S. C.; FITCH, R. J.; BRUNNER, R. L.; SPARKES, R. S. Dopamine receptor gene and cigarette smoking: a reward gene? Medical Hypotheses, Edinburgh, v. 42, n.4, p. 257-260, Apr. 1994.

NUNES, S. O. V.; ONISHI, L. O.; HASHIMOTO, S. M., KIKUCHI, R.; TOLEDO, L. G. M.; KOIKE, A.; CARMO, D. R.; PAOLIELO, M. M. B.; MATSUO, T. A história familial e a prevalência de dependência de álcool e tabaco em área metropolitana na região Sul do Brasil. Revista de Psiquiatria Clínica, São Paulo, v. 26, n. 3, p. 84-89, maio/jun. 1999.

ORGANIZAÇÃOMUNDIALDASAÚDE. Classificação de transtornos mentais e de comportamento da CID-10: critérios diagnósticos para pesquisa. Porto Alegre: Artes Médicas, 1998.

PELUCCHI, C.; GALLUS, S.; GARAVELLO, W.; BOSETTI, C.; LA VECCHIA, C. Cancer risk associated with alcohol and tobacco use: focus on upper aero digestive tract and liver. Alcohol Research \& Health, Rockville, v. 29, n.3, p. 193-198, 2006.

SCHMITZ, N.; KRUSE, J.; KUGLER, J. Disabilities, quality of life, and mental disorders associated with smoking and nicotine dependence. The American Journal of Psychiatry, Arlington, v. 160, n. 9, p. 16701676, 2003.

WORLD HEALTH ORGANIZATION. The alcohol, smoking and substance involvement screening test (ASSIST): guidelines for use in primary care - Draft version 1.1 for field testing. Geneva: World Health Organization, 2003. Disponível em: <http://www.who. int/substance_abuse/activities/en/Draft_The_ASSIST_ Guidelines.pdf $>$. Acesso em: 6 abr. 2007. 
